\title{
A Quasi-Monostatic Reflective Three-Antenna Method for Antenna Gain and Scattering Measurement
}

\author{
Hsin-Chia Lu* and Tah-Hsiung Chu \\ Department of Electrical Engineering \\ National Taiwan University, Taipei, Taiwan, R.O.C. \\ E-mail:leonardo@ew.ee.ntu.edu.tw, thc@ew.ee.ntu.edu.tw
}

\section{INTRODUCTION}

Three-antenna method [1] is a well-known technique to measure the antenna gain without using a reference antenna. However, it operates in a transmission-type arrangement, a long return cable is then required. Several methods were developed to eliminate the return cable by using the radar cross section (RCS) measurement approach. The advantages of this approach are described in [2]-[6]. In [7], a method based on the RCS measurement concept and antenna scattering matrix is developed. It can not only measure the antenna gain, but also derive the structural scattering characteristics and antenna input impedance from the measurement. However, it may require polarimetric calibration procedure for the measurement system.

In the last year, we proposed a method [8] that combines the concepts of threeantenna method and RCS measurement method to measure the antenna gain and its structural scattering characteristics without involving the reference antenna or polarimetric calibration. However, the measurement sensitivity may be limited by the input mismatch of transmitting antenna.

In this paper, we propose a quasi-monostatic arrangement that will greatly improve the measurement sensitivity. Three antennas are arranged as transmitting, receiving and reflecting antennas as shown in Fig. 1, which is represented as a three-port network. By measuring the two-port scattering parameters at the reference planes of transmitting and receiving antennas while the reflecting antenna is terminated with three different terminators, the three-port scattering parameters can be calculated. One can then follow the three-antenna method to find the gain of each antenna. In addition, the structural scattering characteristics of each antenna can be solved.

\section{FORMULATION}

As shown in Fig. 1, antennas $i, j$ and $k$ and the free space propagation terms between them can be considered as a three-port network with its reference planes at the terminating ports $i, j$ and $k$. The three-port scattering matrix $\left[S^{T}\right]$ of this three-port network is hence related to the antenna scattering matrices of these three antennas. The formulation to derive the three-port scattering matrix $\left[S^{T}\right]$ using the two-port measurement is given in the following Sec. II.A. The relation between the three-port scat- 
tering parameters $\left[S^{T}\right]$ and the three antenna scattering matrices is then given in Sec. II.B. Finally, the concept of three-antenna method is adopted to give the gain and structural scattering of each antenna.

A. Three-port scattering parameter measurement using two-port network analyzer

By connecting three known different terminators $\Gamma_{k_{1}}, \Gamma_{k_{2}}$ and $\Gamma_{k_{3}}$ at the port $k$ of a reciprocal three-port network, the measured two-port scattering parameters are related to the original three-port scattering parameters as

$$
\begin{aligned}
& S_{i j}^{\left(k_{1}\right)}=S_{i j}+\frac{S_{i k} S_{k j} \Gamma_{k_{1}}}{1-S_{k k} \Gamma_{k_{1}}}, \\
& S_{i j}^{\left(k_{2}\right)}=S_{i j}+\frac{S_{i k} S_{k j} \Gamma_{k_{2}}}{1-S_{k k} \Gamma_{k_{2}}}, \\
& S_{i j}^{\left(k_{3}\right)}=S_{i j}+\frac{S_{i k} S_{k j} \Gamma_{k_{3}}}{1-S_{k k} \Gamma_{k_{3}}} .
\end{aligned}
$$

By equating $S_{i k} S_{k j}$ at the right hand side in (1) to (3), one can obtain two linear equations of $S_{i j}$ and $S_{k k}$ as

$$
\begin{aligned}
& \left(\frac{1}{\Gamma_{k_{1}}}-\frac{1}{\Gamma_{k_{2}}}\right) S_{i j}+\left(S_{i j}^{\left(k_{1}\right)}-S_{i j}^{\left(k_{2}\right)}\right) S_{k k}=\frac{S_{i j}^{\left(k_{1}\right)}}{\Gamma_{k_{1}}}-\frac{S_{i j}^{\left(k_{2}\right)}}{\Gamma_{k_{2}}}, \\
& \left(\frac{1}{\Gamma_{k_{1}}}-\frac{1}{\Gamma_{k_{3}}}\right) S_{i j}+\left(S_{i j}^{\left(k_{1}\right)}-S_{i j}^{\left(k_{3}\right)}\right) S_{k k}=\frac{S_{i j}^{\left(k_{1}\right)}}{\Gamma_{k_{1}}}-\frac{S_{i j}^{\left(k_{3}\right)}}{\Gamma_{k_{3}}} .
\end{aligned}
$$

From (4) and (5), $S_{i j}$ and $S_{k k}$ can be solved. In addition, $S_{i k} S_{k j}$ can be calculated by substituting the resulted $S_{i j}$ and $S_{k k}$ into (1) to (3). In addition, one can calculate $S_{i i}, S_{i k} S_{k i}$ and $S_{j j}, S_{j k} S_{k j}$ from measured two-port scattering parameters.

\section{B. Quasi-monostatic reflective three-antenna method}

In the following derivation, each antenna is represented as a two-port network, denoted by its terminating port and radiation port as shown in Fig.1(a). This two-port scattering matrix describes the antenna input impedance, structural scattering and transmitting and receiving characteristics [7]. Taking antenna $i$ in Fig. 1 for example, it is given as

$$
\left[\begin{array}{l}
b_{1}^{i} \\
b_{2}^{i}
\end{array}\right]=\left[\begin{array}{ll}
S_{11}^{i} & S_{12}^{i} \\
S_{21}^{i} & S_{22}^{i}
\end{array}\right]\left[\begin{array}{l}
a_{1}^{i} \\
a_{2}^{i}
\end{array}\right]
$$

In [6], $S_{11}^{i}$ is the antenna input impedance, $S_{12}^{i}=S_{21}^{i}$ accounts for the antenna transmitting or receiving characteristics, and $S_{22}^{i}$ describes the antenna structural scattering 
characteristics. Based on the formulation given in Sec. II.A, the scattering matrix $\left[S^{T}\right]$ can be calculated from three reflection measurements by terminating port $k$ with three different terminators. $\left[S^{T}\right]$ is a three-port network and it can be shown that it is related to the properties of antennas $i, j$ and $k$ and free space propagation as

$$
\begin{gathered}
S_{i i}=S_{11}^{i}+\frac{\left(S_{12}^{i}\right)^{2} S_{22}^{k} T^{2}}{M}, S_{j j}=S_{11}^{j}+\frac{\left(S_{12}^{j}\right)^{2} S_{22}^{k} T^{2}}{M} \\
S_{k k}=S_{11}^{k}+\frac{\left(S_{12}^{k}\right)^{2} S_{22}^{i} T^{2}+\left(S_{12}^{k}\right)^{2} S_{22}^{j} T^{2}}{M} \\
S_{i j}=S_{j i}=\frac{S_{12}^{i} S_{22}^{k} S_{12}^{j} T^{2}}{M}, S_{k i}=\frac{S_{12}^{i} S_{12}^{k} T}{M}, S_{j k}=\frac{S_{12}^{j} S_{12}^{k} T}{M}
\end{gathered}
$$

where $T=e^{-j k R} /(\sqrt{4 \pi} R)$ is the range term, and $M=1-S_{22}^{i} S_{22}^{k} T^{2}-S_{22}^{j} S_{22}^{k} T^{2}$.

As the input impedance of each antennas is measured in advance, one can calculate $\Delta S_{i i}, \Delta S_{j j}$ and $\Delta S_{k k}$ as $\Delta S_{i i}=S_{i i}-S_{11}^{i}, \Delta S_{j j}=S_{j j}-S_{11}^{j}$ and $\Delta S_{k k}=S_{k k}-S_{11}^{k}$. The denominator $M$ in (7) to (9) can then be calculated as

$$
M=1-\frac{\Delta S_{i i} \Delta S_{j j} \Delta S_{k k}}{S_{i j} S_{k i} S_{j k}}
$$

Hence, the product of the transmitting characteristics of three antennas becomes

$$
S_{12}^{i} S_{12}^{j}\left(S_{12}^{k}\right)^{2}=S_{j k} S_{k i} \frac{M^{2}}{T^{2}} .
$$

In (11), $S_{12}^{k}$ term is squared because antenna $k$ is the reflecting antenna. As each antenna is used as the reflecting antenna, $\left(S_{12}^{i}\right)^{2} S_{12}^{j} S_{12}^{k}$ and $S_{12}^{i}\left(S_{12}^{j}\right)^{2} S_{12}^{k}$ can be acquired. Therefore, similar to the three-antenna method, one can solve the transmitting characteristics of each antenna. Note there are four possible sets of solutions separated by $90^{\circ}$ for the transmission characteristics. In our previous method [8], the product of gain depends on the reflection measurement of the transmitting antenna, the sensitivity is then limited. While in this quasi-monostatic arrangement, the product of transmission characteristics in (11) depends on the transmission measurement between transmitting and receiving antennas, i.e., $S_{i j}^{(k)}$. Therefore, the measurement sensitivity can be improved.

As $S_{12}^{i}$ is solved, the maximum available antenna gain $G_{i}^{m}$ and the transducer antenna gain $G_{i}^{t}$ of the antenna $i$ can be calculated as

$$
G_{i}^{m}=\frac{\sqrt{4 \pi}}{\lambda} \frac{\left|S_{12}^{i}\right|^{2}}{1-\left|S_{i 1}^{i}\right|^{2}} \text { and } G_{i}^{i}=\frac{\sqrt{4 \pi}}{\lambda}\left|S_{i 2}\right|^{2} \text {. }
$$


Since only the absolute value of $S_{12}^{i}$ is involved in the antenna gain calculation, its phase ambiguity is not important. The structural scattering characteristics of antenna $k$ is then given as $S_{22}^{k}=\Delta S_{i i} \frac{M}{\left(S_{12}^{i}\right)^{2} T^{2}}$.

\section{CONCLUSION}

In this paper, we propose a novel approach to measure the antenna gain and structural scattering characteristics using a quasi-monostatic reflective three-antenna method. This method can eliminate the needs of a return cable, reference antenna or the polarimetric calibration procedure. In addition, the sensitivity of measurement arrangement can be improved.

IV. REFERENCE

[1] J. S. Hollis, T. J. Lyon and L. Clayton, Microwave Antenna Measurements, Ch.8, Scientific-Atlanta Inc. 1970

[2] D. D. King, "Measurement and interpretation of antenna scattering," Proc. IRE., vol. 37, pp. $770-777$, July 1949

[3] R. J. Garbacz, "Determination of antenna parameters by scattering cross section measurements," Proc. Inst. Elect. Eng., vol. 111, no. 10, pp. 1679-1686, Oct. 1964.

[4] J. Appel-Hansen, "Accurate determination of gain and radiation patterns by radar crosssection measurements," IEEE Trans. Antennas Propagat., vol. AP-27, pp. 640-646, Sept. 1979.

[5] J. J. H. Wang, C. W. Choi and R. L. Moore, "Precession experimental characterization of the scattering and radiation properties of antennas," IEEE Trans. Antennas Propagat, vol. AP-30, pp. 108-112, Jan. 1982

[6] K. M. Lambert, R. C. Rudduck and T. H. Lee, "A new method for obtaining antenna gain from backscatter measurements," IEEE Trans. Antennas Propagat., vol. AP-38, pp.896902 , June 1990

[7] W. Wiesbeck and E. Heidrich, "Wide-band multiport antenna characterization by polarimetric RCS measurement," IEEE Trans. Antennas Propagat., vol. AP-46, pp. 341-350, March 1998.

[8] H. C. Lu and T. H. Chu, "Antenna gain and structural scattering measurement using reflective three-antenna method," 1999 IEEE AP-S International Symposium and URSI Radio Science Meeting, pp. 374-377, July 1999.

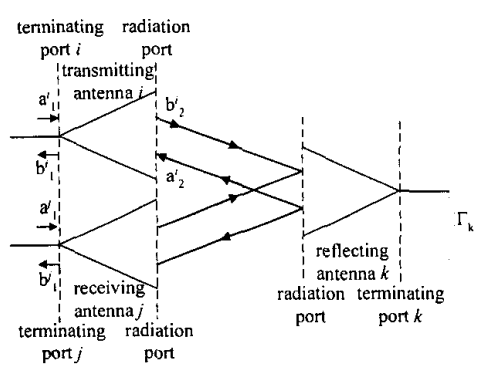

(a)

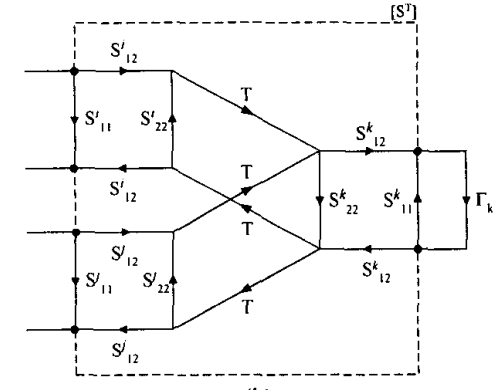

(b)

Fig. 1 (a) Schematic diagram of quasi-monostatic reflective three-antenna method and (b) its scattering parameter representation. 